\title{
ASYMPTOTIQUE DU NOMBRE DE RÉSONANCES DE L'OPÉRATEUR DE SCHRÖDINGER AVEC POTENTIEL LINÉAIRE ET MATRICIEL
}

\section{NEDELEC}

\section{Introduction}

Dans ce travail nous étudions les propriétés spectrales des opérateurs de Schrödinger à potentiels matriciels. De tels opérateurs apparaissent dans l'approximation de Born-Oppenheimer, qui est developpée par exemple dans les travaux de Hagedorn [6] de Klein, Martinez, Sailer et Wang [10] et de Combes, Duclos et Seiler [3].

Notre travail se consacre en particulier à l'étude des résonances. Les résonances qui sont associées en physique à des états quasi-stables, sont introduites rigoureusement dans le travail de Aguilar et Combes [1], et de manière plus générale par Hunziker [9] ou par Helffer et Martinez [7].

Plus précisément, pour ce faire une idée de la répartition des résonances, nous étudions la fonction de comptage, qui est le nombre de résonances de ces opérateurs dans une boule de rayon donné.

L'étude du comportement asymptotique de la fonction de comptage d'opérateurs de Schrödinger à fait l'objet de nombreux travaux, mais la plupart d'entre eux se limitent à l'obtention de majorants. Toutefois quelques résultats plus complets ont étés obtenus récemment avec l'obtention de minorants et parfois d' équivalents. Guillopé et Zworski [5] obtiennent une borne inférieure polynomiale et optimale dans le cas d'opérateurs de Laplace sur des surfaces de Riemann. Zworski obtient des résultats plus précis pour des opérateurs à symétrie radiale dans [17] et pour des opérateurs dans des espaces de petite dimension [16]. Par ailleurs Sjöstrand et Zworski obtiennent des minorations pour des perturbations trés générales du Laplacien dans [14] [13]. Dans ces derniers articles, la démonstration s'appuie sur la comparaison des opérateurs étudiés à des opérateurs modèles connus. Cette comparaison est développée dans les travaux de Vodev [15] et de Sjöstrand et Zworski [13].

Dans un travail précédent [11] nous avions déja étudié des opérateurs de Schrödinger à potentiel matriciel linéaire. L' étude de tels opérateurs est intéressante car elle fait apparaître des résonances d'un type inusuel. En effet, l'existence des résonances ne s'explique pas par la présence de trajectoires captées, comme c'est en général le cas, mais par le croisement transversal des valeurs propres de la matrice du potentiel. Nous avions mis en évidence ce phénomène

Received January 13, 1997. 
dans [11] et nous avions montré en sus que le spectre d'un opérateur matriciel non linéaire pouvait se comparer aisément à celui d'un opérateur à potentiel matriciel linéaire. Dans l'article ici présent nous nous limiterons donc au cas de potentiels matriciels linéaires.

Notre principal résultat est l'obtention de l'asymptotique de la fonction de comptage de l'opérateur de Schrödinger avec potentiel linéaire et matriciel au voisinage de l'infini.

L'article est organisée comme suit. Dans la section 2 nous précisons les hy-

pothèses et nous énonçons notre résultat. Dans la section 3 nous citons des exemples de potentiels qui vérifient les hypothèses. Pour les besoins de la démonstration, un calcul symbolique et un calcul fonctionnel analytique sont respectivement introduit dans les sections 4 et 5 . Dans la section 6 , une majoration de la fonction de comptage est obtenue à l'aide des inégalités de Weyl. La minoration est obtenue dans la section 7 , en utilisant une formule de trace.

\section{Résultats et Notations}

Soit $x \in \mathbb{R}^{n}$. Considérons une application linéaire $x \rightarrow V(x)$ de $\mathbb{R}^{n}$ dans l'ensemble des matrices hermitiennes à valeurs dans $\mathbb{C}$ de taille $(r, r)$.

Notons $P$ l'opérateur $P=-\Delta \otimes I_{r}+V(x)$. Notons (H0) la proposition : Il existe une constante $C>0$ telle que :

$$
\forall x \in \mathbb{R}^{n} \quad \forall u \in \mathbb{R}^{r} \quad \frac{1}{C}\||x| u\| \leq\|V(x) u\| \leq C\||x| u\| .
$$

Les résonances de l'opérateur $P$ sont définies lorsque la matrice $V(x)$ vérifie l'hypothèse (Ho) dans [11]. Notons $N(r)$ le nombre de résonances de $P$ de module inférieur à $r$. Le résultat obtenu est le suivant.

Théorème 2.1. Si $V$ vérifie $\mathbf{( H 0 )}$ alors, il existe une constante $C>0$ telle que

$$
N(r) \leq C r^{\frac{3 n}{2}}
$$

Si de plus $n$ est pair, il existe une constante $C>0$ telle que

$$
\frac{1}{C} r^{\frac{3 n}{2}} \leq N(r) \leq C r^{\frac{3 n}{2}}
$$

Rappel sur les résonances : Notons, pour $\theta \in] 0, \frac{\pi}{3}\left[, P_{\theta}=e^{-2 i \theta} \Delta+e^{i \theta} V(x)\right.$. Le domaine de $P_{\theta}$ est $\mathcal{D}=\left\{u \in H^{2}\left(\mathbb{R}^{n}\right),|x| u \in L^{2}\right\}$. Les opérateurs $P_{\theta}$ ont un spectre discret indépendant de $\theta$. Les résonances sont alors définies comme étant les valeurs propres d'un des opérateurs $P_{\theta}=e^{-2 i \theta} \Delta+e^{i \theta} V(x)$ pour $\left.\theta \in\right] 0, \frac{\pi}{3}[$. Elles appartiennent au secteur $\left\{z, \operatorname{Im}\left(z e^{-i \theta}\right) \leq 0\right\}$ [11] et donc à l'intersection de ces secteurs lorsque $\theta$ varie. 


\section{Exemples d'opérateurs $V$}

Dans cette section nous donnons quelques exemples de potentiels vérifiant l'hypothèse (H0). Remarquons que l'hypothèse (H0) implique que le polynôme homogène de degré $r$ défini par $x \rightarrow \operatorname{det} V(x)$ ne s'annule qu'en 0 . Ceci n'est possible que si $n=1$ ou si $r$ est pair.

Si $n=1$ alors les matrices $V$ vérifiant (H0) sont de la forme $V(x)=x V_{0}$ où $V_{0}$ est une matrice hermitienne constante admettant $r$ valeurs propres distinctes de zéro.

Déterminons toutes les matrices $V(x)$ linéaires en $x$, hermitiennes et vérifiant (H0) lorsque $r=2$ et $n>1$. On peut toujours écrire $V$ sous la forme :

$$
V(x)=\operatorname{tr} V(x)+\left(\begin{array}{ll}
y_{1}(x) & y_{2}(x)+i y_{3}(x) \\
y_{2}(x)-i y_{3}(x) & -y_{1}(x)
\end{array}\right),
$$

où les $y_{1}, y_{2}$ et $y_{3}$ sont des formes linéaires réelles. Les valeurs propres de $V(x)$ sont alors $\operatorname{tr} V(x) \pm \sqrt{\sum_{j \in\{1,2,3\}} y_{j}^{2}(x)}$. L'hypothèse (Ho) est alors équivalente à l'existence d'une constante $C>0$ telle que

$$
\left|\operatorname{tr} V(x) \pm \sqrt{\sum_{j \in\{1,2,3\}} y_{j}^{2}(x) \mid} \geq \frac{1}{C}\right| x \mid .
$$

Supposons que les $\left(y_{j}\right)_{j \in\{1,2,3\}}$ forment un système de rang inférieur strictement à $n$. Alors l'une des valeurs propres de $V(x)$ s'annule en un point $x_{0}$ différent de 0 . Ceci contredit l'hypothèse (Ho).

Supposons que les $\left(y_{j}\right)_{j \in\{1,2,3\}}$ forment un système de rang $n$. Alors (1) implique

$$
\left\{\begin{array}{l}
\sqrt{\sum_{j \in\{1,2,3\}} y_{j}^{2}(x)}+\operatorname{tr} V(x) \geq C^{\prime}|x| \\
\sqrt{\sum_{j \in\{1,2,3\}} y_{j}^{2}(x)}-\operatorname{tr} V(x) \geq C^{\prime}|x|
\end{array} \quad \forall x \neq 0 .\right.
$$

Cette contrainte s'écrit aussi

$$
|\operatorname{tr} V(x)| \leq C \sqrt{\sum_{j \in\{1,2,3\}} y_{j}^{2}(x)}
$$

avec $C<1$ et sous cette condition (Ho) est vérifiée.

Si $r=4$, un exemple de matrice vérifiant les hypothèses est donné par

$$
\begin{aligned}
V(x) & =y_{6}(x)+ \\
& \left(\begin{array}{llll}
y_{1}(x) & 0 & y_{2}(x)+i y_{3}(x) & y_{4}(x)+i y_{5}(x) \\
0 & y_{1}(x) & -y_{4}(x)+i y_{5}(x) & y_{2}(x)-i y_{3}(x) \\
y_{2}(x)-i y_{3}(x) & -y_{4}(x)-i y_{5}(x) & -y_{1}(x) & 0 \\
y_{4}(x)-i y_{5}(x) & y_{2}(x)+i y_{3}(x) & 0 & -y_{1}(x)
\end{array}\right)
\end{aligned}
$$

avec $x \in \mathbb{R}^{n}$, avec $n \leq 5, y_{1}, y_{2}, y_{3}, y_{4}, y_{5}$ et $y_{6}$ sont des formes linéaires réelles, $y_{1}, y_{2}, y_{3}, y_{4}$ et $y_{5}$ forment un système de rang $n$ et de plus il existe une constante 
$C<1$ telle que $\left|y_{6}(x)\right| \leq C\left|y_{1}(x)^{2}+y_{2}(x)^{2}+y_{3}(x)^{2}+y_{4}(x)^{2}+y_{5}(x)^{2}\right|^{\frac{1}{2}}$. Les valeurs propres de cette matrice sont les fonctions :

$$
y_{6}(x) \pm \sqrt{\sum_{j \in\{1,2,3,4,5\}} y_{j}^{2}(x)} .
$$

\section{Calcul symbolique}

Soit $x \in \mathbb{R}^{n}, \xi \in \mathbb{R}^{n}, z \in \mathbb{C}$ et $h \in \mathbb{R}$. Une fonction $f(x, \xi, z)$ de $\mathbb{R}^{2 n} \times \mathbb{C}$ dans $\mathbb{R}$ est dite tempérée s'il existe $N>0$ et $C>0$ tels que

$$
\forall(x, \xi, u, v, z) \in \mathbb{R}^{4 n} \times \mathbb{C}|f(x+u, \xi+v, z)| \leq C|f(x, \xi, z)|\left(1+u^{2}+v^{2}\right)^{N} .
$$

Soient $m_{1}(x, \xi, z), m_{2}(x, \xi, z), \varphi(x, \xi, z)$ et $\phi(x, \xi, z)$ quatres fonctions tempérées telles que $\varphi \geq 1$ et $\phi \geq 1$.

Une fonction $f(x, \xi, z)$ de $\mathbb{R}^{2 n} \times \mathbb{C}$ dans $\mathbb{R}$ est dite continue par rapport à $\varphi$ et $\phi$ si il existe une constante $C>0$ telle que :

$$
\begin{gathered}
\text { pour tous }(x, \xi, u, v, z) \in \mathbb{R}^{4 n} \times \mathbb{C} \text { vérifiant } \frac{u^{2}}{\varphi^{2}(x, \xi, z)}+\frac{v^{2}}{\phi^{2}(x, \xi, z)} \leq \frac{1}{C} \text { alors } \\
\frac{1}{C} f(x, \xi, z) \leq f(x+u, \xi+v, z) \leq C f(x, \xi, z) .
\end{gathered}
$$

Supposons que les fonctions $m_{1}(x, \xi, z), m_{2}(x, \xi, z) \varphi(x, \xi, z)$ et $\phi(x, \xi, z)$ sont continues par rapport à $\varphi$ et $\phi$. Soit $\Omega$ un sous ensemble de $\mathbb{R}^{2 n} \times \mathbb{C}$.

Notons $\Sigma(\Omega \times] 0, h_{0}[, m, \varphi, \phi)$ l'ensemble des symboles $a \in \mathcal{C}^{\infty}(\Omega)$ à valeurs dans $\mathcal{M}_{r}(\mathbb{C})$ tels que pour tout $(\alpha, \beta) \in \mathbb{N}^{2 n}$, il existe $C_{\alpha, \beta}$ telle que

$$
\forall(x, \xi, z, h) \in \Omega \times] 0, h_{0}\left[, \quad\left\|\partial_{x}^{\alpha} \partial_{\xi}^{\beta} a(x, \xi, z, h)\right\| \leq C_{\alpha, \beta}\left(m \varphi^{-\alpha} \phi^{-\beta}\right)(x, \xi, z) .\right.
$$

Lorsque le symbole $a$ ne dépend pas du paramètre $h$ on remplace l'ensemble $\Omega \times] 0, h_{0}[$ par $\Omega$. Un symbole $a$ est dit $h$-admissible s'il existe des symboles $a_{j} \in \Sigma\left(\Omega, \frac{m}{\varphi^{j} \phi^{j}}, \varphi, \phi\right)$ tels que pour tout $N$ :

$$
a(x, \xi, z, h)=\sum_{j<N} h^{j} a_{j}(x, \xi, z)+h^{N} a_{N}^{\prime}(x, \xi, z, h),
$$

avec $a_{N}^{\prime} \in \Sigma(\Omega \times] 0, h_{0}\left[, \frac{m}{\varphi^{N} \phi^{N}}, \varphi, \phi\right)$. Soit $S$ tel que $\mathbb{R}^{2 n} \times S \subset \Omega$. Au symbole $a h$-admissible on associe un opérateur dépendant du paramètre $z \in S$ par la formule

$$
\left(\mathrm{Op}^{w}(a)(z) u\right)(x)=(2 \pi h)^{-n} \int_{\mathbb{R}^{2 n}} e^{i\langle x-y, \xi\rangle / h} a\left(\frac{x+y}{2}, \xi, z\right) u(y) d y d \xi .
$$

Le calcul de Weyl [8], prouve la proposition suivante. 
Proposition 4.1. Si $a \in \Sigma\left(\mathbb{R}^{2 n} \times S \times\right] 0, h_{0}\left[, m_{1}, \varphi, \phi\right)$ et $b \in \Sigma\left(\mathbb{R}^{2 n} \times S \times\right] 0$, $h_{0}\left[, m_{2}, \varphi, \phi\right)$ sont deux symboles $h$-admissibles, holomorphes en $z$, alors pour tout $N$, il existe deux symboles $c$ et $d$ tels que pour tout $z \in S$,

$$
\mathrm{Op}^{w}(a)(z) \mathrm{Op}^{w}(b)(z)=\mathrm{Op}^{w}(c)(z)+h^{N} \mathrm{Op}^{w}(d)(z),
$$

où $c \in \Sigma\left(\mathbb{R}^{2 n} \times S \times\right] 0, h_{0}\left[, m_{1} m_{2}, \varphi, \phi\right)$ est un symbole $h$-admissible holomorphe en $z$ et $d$ est un symbole de $\Sigma\left(\mathbb{R}^{2 n} \times S \times\right] 0, h_{0}\left[, \frac{m_{1} m_{2}}{\varphi^{N} \phi^{N}}, \varphi, \phi\right)$. Le développement asymptotique de c est donnée par

$$
\begin{gathered}
c(x, \xi, z)=\sum_{j<N} h^{j} c_{j}(x, \xi, z), \text { avec } \\
c_{j}(x, \xi, z)=\frac{1}{2^{j}} \sum_{|\alpha+\beta|=j} \frac{(-1)^{|\beta|}}{\alpha ! \beta !}\left(\partial_{\xi}^{\alpha} D_{x}^{\beta} a\right)\left(\partial_{\xi}^{\beta} D_{x}^{\alpha} b\right)(x, \xi, z) .
\end{gathered}
$$

Les propriétés des symboles $a$ et $b$ se répercutent sur $c$.

Proposition 4.2. Si $a \in \Sigma(\Omega \times] 0, h_{0}\left[, m_{1}, \varphi, \phi\right)$ et $b \in \Sigma(\Omega \times] 0, h_{0}\left[, m_{2}, \varphi, \phi\right)$ alors le symbole c donné par la formule (2) appartient à $\Sigma(\Omega \times] 0, h_{0}\left[, m_{1} m_{2}, \varphi, \phi\right)$.

\section{Calcul fonctionnel}

Soit $c_{0} \in \mathbb{R}^{+}$. Introduisons les opérateurs semi-classiques

$$
P^{h}=-h^{2} \Delta \otimes I_{r}+V(x), \quad P_{\theta}^{h}=-h^{2} e^{-2 i \theta} \Delta \otimes I_{r}+e^{i \theta} V(x),
$$

et l'opérateur $Q^{h}=e^{i \frac{\pi}{3}} P_{\frac{\pi}{6}}^{h}+c_{0}$. Soit $r \in \mathbb{R}^{+}$. Notons $N^{h}(r)$ le nombre de résonances de l'opérateur $P^{h}$ de module inférieur ou égal à $r$. Comme l'opérateur $P_{\theta}^{h}$ est unitairement équivalent à $\lambda P_{\theta}^{h \lambda^{-3 / 2}}$, on trouve :

$$
N^{h}(r)=N^{1}\left(r h^{-\frac{2}{3}}\right)=N^{h r^{-\frac{3}{2}}}(1) \text {. }
$$

Notons $M^{h}(r)$ le nombre de valeurs propres de $Q^{h}$ de module inférieur à $r$. En comparant les valeurs propres de $Q^{h}$ et les résonances de $P^{h}$, on prouve que

$$
N^{h}\left(r-c_{0}\right) \leq M^{h}(r) \leq N^{h}\left(r+c_{0}\right)
$$

Le symbole semi-classique de $Q^{h}$ est donné par $q(x, \xi)=\xi^{2}+i V(x)+c_{0}$.

Notons $\mathcal{P}^{-}=\left\{z \in \mathbb{C}, \quad \operatorname{Re}\left(z-c_{0}\right)<0\right\}$. Soit $f$ une fonction vérifiant les hypothèses (H1) et (H2) :

(H1) $f$ est holomorphe sur $\mathbb{C} \backslash S^{-}$où $S^{-}$est un cône inclus strictement dans $\mathcal{P}^{-}$, que l'on supposera de la forme $S^{-}=\{z,|\operatorname{Im}(z)| \leq-C \operatorname{Re} e(z)\}$ où $C$ est une constante telle que $C>0$.

(H2) Il existe $k \in \mathbb{R}$ et $c \in \mathbb{R}$ tels que $f$ vérifie la relation

$$
\forall z \in \mathbb{C} \backslash S^{-} \text {et }|z|>c \text { alors }|f(z)| \leq \frac{C}{|z|^{k}} .
$$


Soit $\tilde{S}$ un cône contenant $S^{-}$de la forme

$$
\tilde{S}=\left\{z,|\operatorname{Im}(z)| \leq-\tilde{C}\left(\mathrm{R} e(z)-\frac{c_{0}}{2}\right)\right\},
$$

où $\tilde{C}>C$. D'après l'article [11], pour $z \in \tilde{S}$, l'opérateur $Q-z$ est inversible et il existe une constante $C>0$ telle que

$$
\left\|(Q-z)^{-1}\right\|_{\mathcal{L}\left(L^{2}, D\right)} \leq C,\left\|(Q-z)^{-1}\right\|_{\mathcal{L}\left(L^{2}, L^{2}\right)} \leq C|z|^{-1}
$$

On peut définir alors l'opérateur $f\left(Q^{h}\right)$ à l'aide du calcul fonctionnel par la formule standard

$$
f\left(Q^{h}\right)=\frac{1}{2 i \pi} \int_{\gamma_{0}} f(z)\left(z-Q^{h}\right)^{-1} d z
$$

pour $k>1$, où $\gamma_{0}$ est un contour inclus dans $\mathcal{P}^{-}$, entourant le cône $S^{-}$et inclus dans le cône $\tilde{S}$ orienté du haut vers le bas.

La fonction $(x, \xi, z) \rightarrow\left(1+|x|+\xi^{2}+|z|\right)=m(x, \xi, z)$ est tempérée, il existe une constante $C>0$ telle que

$$
\frac{1}{C} m(x, \xi, z) \frac{1}{1+|u|+v^{2}} \leq m(x+u, \xi+v, z) \leq C m(x, \xi, z)\left(1+|u|+v^{2}\right) .
$$

Posons $\varphi=m$ et $\phi=m^{\frac{1}{2}}$. La fonction $m$ est continue par rapport à $\varphi$ et $\phi$. Notons :

$$
\Omega=\left\{(x, \xi, z) \in \mathbb{R}^{2 n} \times \mathbb{C}:\left\|\left(q(x, \xi)-z I_{r}\right)^{-1}\right\| \geq C_{\Omega}\left(1+|x|+\xi^{2}\right)^{-1}\right\} .
$$

L'ensemble $\Omega$ contient $\mathbb{R}^{2 n} \times \tilde{S}$ pour un $C_{\Omega}$ assez grand. Soit $(x, \xi) \in \mathbb{R}^{2 n}$, alors il existe un contour $\gamma_{(x, \xi)} \in \mathbb{C} \backslash S^{-}$qui est une déformation sur un compact du contour $\gamma_{0}$. De plus $\gamma_{(x, \xi)}$ est inclus dans $\left\{z \in \mathbb{C}:|z| \geq\left(1-\frac{1}{C_{\Omega}}\right)\left(1+|x|+\xi^{2}\right)\right\}$ et donc $\left(x, \xi, \gamma_{(x, \xi)}\right) \in \Omega$ (voir Fig. 1).

Le symbole semi-classique $\left(q-z I_{r}\right)$ de $Q^{h}$ appartient à $\Sigma\left(\Omega, m, m, m^{\frac{1}{2}}\right)$. La matrice $\left(q-z I_{r}\right)$ est inversible et $\left(q-z I_{r}\right)^{-1}(x, \xi)$ appartient à $\Sigma\left(\Omega, m^{-1}, m, m^{\frac{1}{2}}\right)$. Etudions le symbole de Weyl de l'opérateur $\left(z I_{r}-Q^{h}\right)^{-1}$. Soit $\tilde{c}_{N}(x, \xi, z)=$ $\sum_{j<N} h^{j} c_{j}(x, \xi, z)$ ou $c_{j}$ est donné par la formule de récurrence :

$$
\begin{aligned}
& c_{j+1}(x, \xi, z)=-c_{0}(x, \xi, z) \times \\
& \sum_{0 \leq l \leq j l+|\alpha|+|\beta|=j+1} \Gamma(\alpha, \beta)\left(\partial_{\xi}^{\alpha} D_{x}^{\beta}\left(q-z I_{r}\right)(x, \xi)\right)\left(\partial_{\xi}^{\beta} D_{x}^{\alpha} c_{l}(x, \xi, z)\right)
\end{aligned}
$$

avec $\Gamma(\alpha, \beta)=\left(\alpha ! \beta ! 2^{|\alpha|}(-2)^{|\beta|}\right)^{-1}$ et $c_{0}(x, \xi, z)=\left(z I_{r}-q(x, \xi)\right)^{-1}$. 


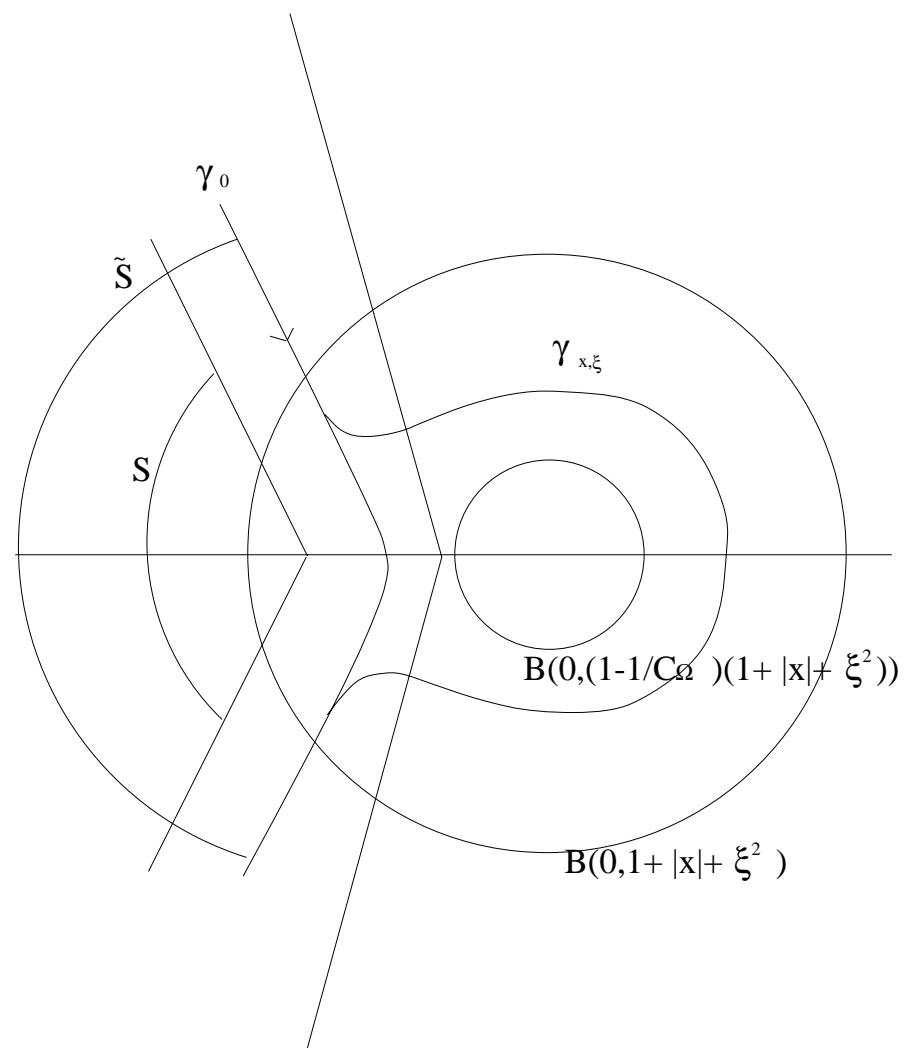

Fig. 1

Le symbole $c_{j}$ appartient à $\Sigma\left(\Omega, m^{-1-j \frac{3}{2}}, m, m^{\frac{1}{2}}\right)$. Le symbole $\tilde{c}_{N}$ est analytique en $z$ sur l'ensemble $\left\{z \in \mathbb{C}: z I_{r} \neq q(x, \xi)\right\}$. Le calcul de $\left(Q-z I_{r}\right) \mathrm{Op}^{w}\left(\tilde{c}_{N}\right)$ donne la formule

$$
\left(Q-z I_{r}\right) \mathrm{Op}^{w}\left(\tilde{c}_{N}\right)=I+h^{N} \mathrm{Op}^{w}\left(d_{N}\right),
$$

avec $d_{N} \in \Sigma\left(\mathbb{R}^{2 n} \times \tilde{S} \times\right] 0, h_{0}\left[, m^{-N \frac{3}{2}}, m, m^{\frac{1}{2}}\right)$. Donc nous obtenons,

$$
\left(Q-z I_{r}\right)^{-1}=\mathrm{Op}^{w}\left(\tilde{c}_{N}\right)-h^{N}\left(Q-z I_{r}\right)^{-1} \mathrm{Op}^{w}\left(d_{N}\right) .
$$

Rappel (Voir [12]) : Un opérateur est à trace dès que son symbole semi-classique $(x, \xi, h) \rightarrow a(x, \xi, h)$ appartient à l'espace :

$$
W^{1, \infty}\left(\mathbb{R}^{2 n}\right)=\left\{a \in L^{1}\left(\mathbb{R}^{2 n}\right), \quad \forall \alpha \in \mathbb{N}^{2 n} \partial^{\alpha} a \in L^{1}\left(\mathbb{R}^{2 n}\right)\right\} .
$$

Sa norme trace est majorée par $C_{n} h^{-n} \sum_{|\alpha| \leq 4 n}\left\|\partial^{\alpha} a(., ., h)\right\|_{L^{1}}$.

L'opérateur $R_{N}(z)=\left(Q-z I_{r}\right)^{-1} \mathrm{Op}^{w}\left(d_{N}\right)$ pour $z \in \tilde{S}$ est un opérateur à trace. Sa norme trace est majorée ,

$$
\exists C>0 \quad\left\|R_{N}(z)\right\|_{t r} \leq C h^{-n} \int_{\mathbb{R}^{2 n}} m(x, \xi, z)^{-N \frac{3}{2}} d x d \xi .
$$


Etudions maintenant le symbole de l'opérateur $f\left(Q^{h}\right)$. Nous avons

$$
f\left(Q^{h}\right)=\frac{1}{2 i \pi} \int_{\gamma_{0}} f(z) \operatorname{Op}^{w}\left(\tilde{c}_{N}\right) d z+\frac{h^{N}}{2 i \pi} \int_{\gamma_{0}} f(z) R_{N}(z) d z
$$

Posons $B_{N}=\frac{1}{2 i \pi} \int_{\gamma_{0}} f(z) \mathrm{Op}^{w}\left(\tilde{c}_{N}\right) d z$ et $\Delta_{N}=\frac{h^{N}}{2 i \pi} \int_{\gamma_{0}} f(z) R_{N}(z) d z$. La norme trace de $\Delta_{N}$ est majorée pour $N$ assez grand par

$$
C h^{N-n} \int_{\mathbb{R}^{2 n} \times \mathbb{R}^{+}} m_{0}(x, \xi)^{-N \frac{3}{2}}(|z|+1)^{-k} d x d \xi d|z|=\mathcal{O}\left(h^{N-n-1}\right) .
$$

Les fonctions $f$ et $\tilde{c}_{N}$ étant holomorphes en $z$, l'opérateur $B_{N}$ a pour symbole

$$
\frac{1}{2 i \pi} \int_{\gamma_{0}} f(z) \tilde{c}_{N}(x, \xi, z) d z=\frac{1}{2 i \pi} \int_{\gamma_{(x, \xi)}} f(z) \tilde{c}_{N}(x, \xi, z) d z
$$

et pour symbole principal $f(q)=\frac{1}{2 i \pi} \int_{\gamma_{0}} f(z) c_{0}(x, \xi) d z$. Majorons la norme $\mathrm{du}$ symbole $\frac{1}{2 i \pi} \int_{\gamma_{(x, \xi)}} f(z) c_{j}(x, \xi, z) d z$ aussi que celles de ces dérivées. En choissisant un bon paramètrage pour le contour $\gamma_{(x, \xi)}$, on prouve :

$$
\left|\frac{1}{2 i \pi} \int_{\gamma_{(x, \xi)}} f(z) c_{j}(x, \xi, z) d z\right|=\mathcal{O}\left(\left(1+|x|+\xi^{2}\right)^{-k-j \frac{3}{2}}\right) .
$$

De même

$$
\left|\partial_{(x, \xi)}^{\alpha} \frac{1}{2 i \pi} \int_{\gamma_{(x, \xi)}} f(z) c_{j}(x, \xi, z) d z\right|=\mathcal{O}\left(\left(1+|x|+\xi^{2}\right)^{-k-j \frac{3}{2}}\right)
$$

L'opérateur $f\left(Q^{h}\right)$ est un opérateur à trace si $\int_{\mathbb{R}^{2 n}}\left(1+|x|+\xi^{2}\right)^{-k} d x d \xi$ converge, ou encore si $k>\frac{3 n}{2}$. La trace de $B_{N}$ est donnée par

$$
(2 \pi h)^{-n} \int_{\mathbb{R}^{2 n}} \operatorname{tr} f(q(x, \xi)) d x d \xi+\mathcal{O}\left(h^{-n+1}\right)
$$

(voir [12] pour les opérateurs à valeurs dans $\mathbb{R}$ et [2] pour la généralisation aux opérateurs à valeurs opérateurs.)

Comme $f\left(Q^{h}\right)=B_{N}+\Delta_{N}$ et $\operatorname{tr} \Delta_{N}=\mathcal{O}\left(h^{N-n-1}\right)$, nous avons pour $N$ assez grand

$$
\operatorname{tr} f\left(Q^{h}\right)=(2 \pi h)^{-n} \int_{\mathbb{R}^{2 n}} \operatorname{tr} f(q(x, \xi)) d x d \xi+\mathcal{O}\left(h^{-n+1}\right) .
$$




\section{Majoration des résonances}

Prenons dans cette section exclusivement pour fonction $f$ la fonction $f(x)=$ $x^{-k}$ avec $k$ un entier pair tel que $k>\frac{3 n}{2}$. Alors la fonction $g(x)=f\left(\frac{1}{x}\right)$ est holomorphe sur $\mathbb{C}$. Elle vérifie $g(x) \geq 0$ pour tout $x \in \mathbb{R}^{+}, g(0)=0, t \rightarrow g\left(e^{t}\right)$ est convexe.

L'opérateur $\left[Q^{h}\right]^{-1}$ est un opérateur borné et compact car l'injection de $D$ dans $L^{2}\left(\mathbb{R}^{n}\right)$ est compacte. Notons alors $s_{j}^{-1}$ les valeurs caractéristiques de $\left[Q^{h}\right]^{-1}$ (Par définition ce sont les valeurs propres de l'opérateur $\left[\left[Q^{h}\right]^{-1^{\star}}\left[Q^{h}\right]^{-1}\right]^{\frac{1}{2}}$.) Notons $\lambda_{j}$ les valeurs propres de l'opérateur $Q^{h}$, les valeurs propres de l'opérateur $\left[Q^{h}\right]^{-1}$ sont les $\frac{1}{\lambda_{j}}$ et elles sont indicées par $j \in \mathbb{N}$. D'après un résultat sur les valeurs caractéristiques [4] nous avons alors

$$
\sum_{j \in \mathbb{N}} g\left(\left|\frac{1}{\lambda_{j}}\right|\right) \leq \sum_{j \in \mathbb{N}} g\left(\frac{1}{s_{j}}\right) .
$$

Soit encore

$$
\sum_{j \in \mathbb{N}} f\left(\left|\lambda_{j}\right|\right) \leq \sum_{j \in \mathbb{N}} f\left(s_{j}\right)
$$

Posons $\tilde{f}(x)=x^{-k / 2}, \tilde{f}$ vérifie l'hypothèse $(\mathbf{H 1})$. De même que pour l'opérateur $f\left(Q^{h}\right)$ on prouve que l'opérateur $\tilde{f}\left(Q^{h} Q^{h^{\star}}\right)$ est à trace. Sa trace est égale à $\sum_{j \in \mathbb{N}} f\left(s_{j}\right)$, ainsi qu'à :

$$
(2 \pi h)^{-n} \int_{\mathbb{R}^{2 n}} \operatorname{tr} f\left(\left(q(x, \xi) q(x, \xi)^{\star}\right)^{\frac{1}{2}}\right) d x d \xi+\mathcal{O}\left(h^{-n+1}\right) .
$$

Ce qui prouve qu'il existe $C>0$ telle que

$$
\sum_{j \in \mathbb{N}} f\left(\left|\lambda_{j}\right|\right) \leq h^{-n} C
$$

Nous allons maintenant exploiter cette relation pour obtenir une majoration de $N^{1}(r)$.

$$
M^{h}(r) f(r)=M^{h}(r) \inf _{s \in[0, r]}(f(s)) \leq \sum_{j \in \mathbb{N}} f\left(\left|\lambda_{j}\right|\right) \leq h^{-n} C .
$$

En utilisant (4) on trouve $N^{h}(c) \leq C h^{-n}$, pour une constante $c>0$. En utilisant (3) on trouve $N^{1}(r) \leq C r^{\frac{3 n}{2}}$.

\section{Minoration du nombre de résonances}

Nous allons obtenir une minoration des résonances dans cette section, pour $n$ pair. Il existe des fonctions localement lipschitziennes $y_{1}(x), y_{2}(x), \cdots y_{r}(x)$, il existe $r$ éléments $\varepsilon_{1}, \varepsilon_{2}, \cdots \varepsilon_{r}$ de $\{1,-1\}$ tels que $y_{i}(x)>0$ pour $x \neq 0, y_{i}(x)$ est positivement homogène de degré 1 , et les valeurs propres de la matrice $V(x)$ sont 
les fonctions $\varepsilon_{i} y_{i}(x)$. D'après le théorème de Lidskii [4] la trace de l'opérateur $f(Q)$ est égale à $\sum_{j \in \mathbb{N}} f\left(\lambda_{j}\right)$, d'après (5) elle est aussi égale à

$$
(2 \pi h)^{-n} \int_{\mathbb{R}^{2 n}} \operatorname{tr} f(q(x, \xi)) d x d \xi+\mathcal{O}\left(h^{-n+1}\right) .
$$

Calculons l'intégrale $I=\int_{\mathbb{R}^{2 n}} \operatorname{tr} f(q(x, \xi)) d x d \xi$. Nous avons

$$
I=\sum_{i \in\{1, \cdots, r\}} \int_{\mathbb{R}^{n} \times \mathbb{R}^{+}} f\left(|\xi|^{2}+i \varepsilon_{i} y_{i}(x)+c_{0}\right)|\xi|^{n-1} d x d|\xi| \operatorname{vol}\left(S^{n-1}\right)
$$

Avec le changement de variable $x=v \frac{w}{y_{i}(w)}, v \in \mathbb{R}^{+}$et $w \in S^{n-1}$ on trouve

$$
\begin{aligned}
I & =\sum_{i \in\{1, \cdots, r\}} \int_{\mathbb{R}^{+2} \times S^{n-1}} f\left(u+i \varepsilon_{i} v+c_{0}\right) u^{\frac{n}{2}-1} v^{n-1} d u d v \frac{d w}{y_{i}(w)^{n}} \frac{\operatorname{vol}\left(S^{n-1}\right)}{2} \\
I & =\sum_{i \in\{1, \cdots, r\}} \frac{\operatorname{vol}\left(S^{n-1}\right)}{2} \int_{S^{n-1}} \frac{d w}{y_{i}(w)^{n}} \int_{\mathbb{R}^{+2}} f\left(u+i \varepsilon_{i} v+c_{0}\right) u^{\frac{n}{2}-1} v^{n-1} d u d v .
\end{aligned}
$$

Calculons l'intégrale $J_{+}=\int_{\mathbb{R}^{+2}} f\left(u+i v+c_{0}\right) u^{\frac{n}{2}-1} v^{n-1} d u d v$ en posant $u+i v=$ $r e^{i \theta}$ avec $r \in \mathbb{R}^{+}$et $\theta \in\left[0, \frac{\pi}{2}\right]$. Nous obtenons

$J_{+}=$

$\int_{\mathbb{R}^{+} \times\left[0, \frac{\pi}{2}\right]}\left(\frac{r}{2} e^{i \theta}\right)^{\frac{3 n}{2}-2}\left(1+e^{-2 i \theta}\right)^{\frac{n}{2}-1}\left(-i\left(1-e^{-2 i \theta}\right)\right)^{n-1} f\left(r e^{i \theta}+c_{0}\right) r e^{i \theta} e^{-2 i \theta} d \theta e^{i \theta} d r$.

En posant $x=e^{-2 i \theta}, r^{\prime}=r e^{i \theta}$, et en utilisant le fait que $f$ est holomorphe on trouve

$$
J_{+}=(-i)^{n} \int_{\mathbb{R}^{+}}\left(\frac{r}{2}\right)^{\frac{3 n}{2}-1} f\left(r+c_{0}\right) d r \int_{[-1,1]}(1+x)^{\frac{1}{2} n-1}(1-x)^{n-1} d x .
$$

Cette intégrale est non nulle pour une fonction $f$ strictement positive sur $\mathbb{R}^{+}$ vérifiant (H2). Elle est réelle si $n$ est pair, purement imaginaire si $n$ est impair. L'intégrale $J_{-}=\int_{\mathbb{R}^{+2}} f\left(u-i v+c_{0}\right) u^{\frac{n}{2}-1} v^{n-1} d u d v$ vaut $J_{+}$si $n$ est pair, $-J_{+}$ si $n$ est impair. Pour $n$ impair, $I$ est purement imaginaire et peut être nulle. Pour $n$ pair, il existe $C>0\left(C=\frac{\operatorname{vol}\left(S^{n-1}\right)}{2} \sum_{i \in\{1, \cdots, r\}} \int_{S^{n-1}} \frac{d w}{y_{i}(w)^{n}}\right)$ telle que $I=C J \neq 0$ et il existe $c>0$ telle que

$$
h^{-n} c \leq\left|\sum_{j \in \mathbb{N}} f\left(\lambda_{j}\right)\right|
$$


Nous allons exploiter cette inégalité pour obtenir une minoration du nombre de résonances de $P^{h}$.

$$
h^{-n} c \leq \sum_{j \in \mathbb{N},\left|\lambda_{j}\right| \leq r}\left|f\left(\lambda_{j}\right)\right|+\sum_{j \in \mathbb{N},\left|\lambda_{j}\right|>r}\left|f\left(\lambda_{j}\right)\right| .
$$

Majorons la quantité $\sum_{j \in \mathbb{N},\left|\lambda_{j}\right|>r}\left|f\left(\lambda_{j}\right)\right|$ à l'aide de la relation (H2). Nous avons

$$
\sum_{j \in \mathbb{N},\left|\lambda_{j}\right| \geq r}\left|f\left(\lambda_{j}\right)\right| \leq \sum_{j \in \mathbb{N},\left|\lambda_{j}\right| \geq r} C\left|\lambda_{j}\right|^{-k} \leq \int_{r}^{\infty} C t^{-k} d M^{h}(t) .
$$

Comme

$$
\int_{r}^{\infty} t^{-k} d M^{h}(t)=k \int_{r}^{\infty} t^{-k-1} M^{h}(t) d t+\left[t^{-k} M^{h}\right]_{r}^{\infty},
$$

de l'inégalité $M^{h}(r) \leq C h^{-n}\left(r+c_{0}\right)^{\frac{3 n}{2}}$, nous déduisons que lorsque $r$ tend vers l'infini :

Soit encore

$$
\sum_{j \in \mathbb{N},\left|\lambda_{j}\right| \geq r}\left|f\left(\lambda_{j}\right)\right|=o_{r}(1) h^{-n}
$$

$$
h^{-n} c \leq M^{h}(r) \sup _{|x| \in\left[\inf \left|\lambda_{j}\right|, r\right]}|f(x)|+h^{-n} o_{r}(1) .
$$

Nous avons pour tout $\lambda_{j}$ valeurs propres de $Q$ la relation $\left|\lambda_{j}\right| \geq c_{0}$. Ceci implique que $\sup _{x \in\left[\inf \left|\lambda_{j}\right|, r\right]}|f(x)|=C>0$. Donc $M^{h}\left(r_{0}\right) \geq h^{-n} c$ pour un $r_{0}$ assez grand. D'après la relation (4) nous avons alors pour un $r_{0}$ assez grand

$$
N^{h}\left(r_{0}\right) \geq h^{-n} c .
$$

La relation (3) implique alors pour tout $r$

$$
N^{1}(r) \geq r^{\frac{3 n}{2}} c
$$

\section{Remerciements}

Je tiens à remercier Johannes Sjöstrand pour ses nombreux conseils.

\section{References}

1. J. Aguilar and J. M. Combes, A class of analytic pertubations for one body Schrödinger Hamiltonians, Comm. Math. Phys. 22 (1971), 269-279.

2. A. Balazard-Konlein. Calcul fonctionnel pour des opérateurs h-admissibles à symbole opérateur et applications, Ph. D. thesis, Université de Nantes, 1985.

3. J. M. Combes, P. Duclos, and R. Seiler, The Born Oppenheimer approximation, Rigorous Atomic and Molecular Physics, 185-212, 1981.

4. I. C. Gohberg and M. G. Krein, Introduction à la théorie des opérateurs linéaires non autoadjoints, Dunod, Paris, 1972.

5. L. Guillopé and M. Zworski, Scattering asymptotics for Riemann surfaces, preprint, 1996.

6. G. A. Hagedorn, Molecular propagation through electron energy level crossings, Memoirs Amer. Math. Soc. 111 (1994). 
7. B. Helffer and A. Martinez, Comparaison entre les diverses notions de résonances, Helv. Phys. Acta 60 (1987), 992-1003.

8. L. Hörmander. The analysis of linear partial differential operators I-IV, Grundlehren Math. Wiss., Springer Verlag, Berlin-New York, 1983.

9. W. Hunziker, Distorsion analyticity and molecular resonances curves, Annales Inst. Henri Poincaré 45 (1986), 339-358.

10. M. Klein, A. Martinez, R. Seiler, and X. P. Wang, On the Born-Oppenheimer expansion for polyatomic molecules, Comm. Math. Phys. 143 (1992), 607-639.

11. L. Nedelec, Résonances semi-classique pour l'opérateur de Schrödinger matriciel en dimension deux, Annales Inst. Henri Poincaré, Physique théorique 65 (1996), 129-162.

12. D. Robert, Autour de l'approximation semi-classique, Prog. Math., Birkhäuser, Boston, 1983.

13. J. Sjöstrand and M. Zworski, Lower bounds on the number of scattering poles, Comm. Partial Diff. Equations 18 (1993), 847-857.

14. L_ Lower bounds on the number of scattering poles,II, J. Funct. Anal. 123 (1994), 336-367.

15. G. Vodev, Asymptotics on the number of scattering poles for degenerate pertubations of the Laplacian, J. Funct. Anal. 138 (1996), 295-310.

16. M. Zworski, Distribution of poles for scattering on the real line, J. Funct. Anal. 73 (1987), 277-296.

17. Sharp polynomial bounds on the number of scattering poles of radial potentials, J. Funct. Anal. 82 (1989), 370-403.

L.A.G.A. Université de Paris Nord, AV. J.B. Clement, F-93430 Villetaneuse, FRANCE

E-mail address: nedelec@ensae.fr 